\title{
Bass Models for EHR, RIS and PACS Diffusion in Finland and Germany
}

\author{
Jens HÜSERS ${ }^{\mathrm{a}, 1}$, Moritz ESDAR ${ }^{\mathrm{a}}$, Maria KUHLMANN ${ }^{\mathrm{a}}$, Kaija SARANTO ${ }^{\mathrm{b}}$, Vesa \\ JORMANAINEN ${ }^{\mathrm{c}}$ and Ursula HÜBNER ${ }^{\mathrm{a}, 1}$ \\ ${ }^{a}$ Health Informatics Research Group, University AS Osnabrück, Germany \\ ${ }^{\mathrm{b}}$ Department of Health and Social Management, University of Eastern Finland \\ ${ }^{c}$ Evaluation of Health Care and Social Welfare Service Provision, Finnish Institute for \\ Health and Welfare, Finland
}

\begin{abstract}
Building on Rogers' Diffusion of Innovation Theory, Bass models describe the diffusion processes distinguishing between innovation ( $p)$ and imitation (q). This study aimed at modelling the uptake of RIS, PACS and EHR systems in Germany and Finland. The Bass models revealed a quick and almost identical uptake process across all three systems for Finland. In contrast, the Bass models mirrored a slower uptake in Germany. Consequently, the Finnish "imitation" coefficients were larger than the German ones. While in Germany almost free market forces were driving the adoption through imitation but without tail wind from policy, the adoption process in Finland was centrally governed. This suggests that the diffusion process in Finland reflected a well-managed roll-out of the systems rather than imitation behaviour. Thus, in order for Bass model coefficients to be understood properly, additional contextual information is required.
\end{abstract}

Keywords. IT-adoption, diffusion, RIS, PACS, EHR, Germany, Finland

\section{Introduction}

The widespread adoption of health IT (HIT) solutions received growing attention of national health policymakers across the globe during the past 20 years. Many countries spawned various eHealth strategies to promote health care digitalization, promising safer and cost-efficient health care delivery. As the various national health systems and the political priorities vary across nations, different strategies have been developed and implemented $[1,2]$. Today, years after the initial political campaigns came - or should have come - to fruition, differing outcomes of their endeavours become apparent. While the Nordic countries are widely recognized to have championed their pursuit of adopting national eHealth infrastructures and solutions early, other countries appeared to be notably less successful in similar undertakings [2]. For instance, Finland had adopted (inter-organisational) electronic health records (EHR) in all national hospitals by 2007, while Germany is still struggling with intra-organisational electronic patient records (EPR) adoption as of today [3]. Although the pioneering role of the Nordic countries has been described extensively in the past, the course of the development, i.e. the diffusion dynamics, have not been modelled statistically. Such models would allow for an in-depth

${ }^{1}$ Corresponding Author, Jens HÜSERS, Health Informatics Research Group, University AS Osnabrück, Germany, E-mail: jenshuesers@hotmail.com. 
understanding by interpreting the model parameters that can be reflected against the background of the national strategies. With longitudinal data on the adoption rates becoming available, it appears valuable to contrast the two countries, i.e. Finland (as an example of a Nordic country) and Germany, with respect to the diffusion of core HIT systems with different complexity. While radiology information systems (RIS) and picture archiving and communication system (PACS) have a clearly described set of functions [4], large-scale EHR implementations are more complex due to the larger spectrum of functions.

The central research purpose was, therefore, to describe and model the uptake of these three key HIT systems to contrast and investigate Germany's and Finland's distinct diffusion dynamics.

\section{Methods}

\subsection{Data sources}

In order to describe and investigate the diffusion of the three selected HIT systems, we used observational data on hospitals obtained in separate national surveys which we then combined to a longitudinal dataset for each country.

The German data stem from the IT-Report Healthcare, an ongoing national research initiative that regularly surveys the digitalization of healthcare delivery organizations, since 2002 [5]. This study uses the results of six separate surveys that were conducted between 2005 and 2017 and addressed German hospitals with CIOs as the primary respondents. In these surveys, hospitals of all ownership and federal states were represented. The data set was post-stratified using demographics from the national hospital register to account for potential selection bias.

The Finnish data stem from national surveys of the Finnish Institute of Health and Welfare (THL) [6] which were commissioned by the Ministry of Social Affairs and Health (MSAH). The data contained hospital adoption rates for the period between 1999 and 2017, providing a total of eight (EHR) and six (RIS and PACS) data points in time.

Due to the well-described functionality, RIS and PACS have more or less clear definitions [7]. In contrast, EHRs, being composed of a multitude of modules, require a precise understanding. By EHRs we mean hospital-wide clinical data repositories that allow for trans-institutional connectivity in general. In the case of Finland, EHRs span across healthcare institutions in a region. In Germany, this may but need not hold true. Thus, in this study, Finland's EHR and Germany's EPR are collectively described as electronic records, and despite the differing meanings, we included both but point out their limited comparability.

\subsection{Data analysis}

Building on Rogers' Diffusion of Innovation Theory [8], the well-established Bass model mathematically describes the diffusion pattern of innovation processes by considering two effects described by its two model coefficients $p$ and $q$, which are interpreted as innovation and imitation in the classical understanding [9]. For each system, i.e. RIS, PACS and electronic records, and country, we fitted a Bass model using a Bayesian approach described in detail in Hüsers et al. [10]. Additionally, the goodness 
of model fit was assessed using $\mathrm{Chi}^{2}$ statistics. Throughout all steps of the analysis, the statistical programming language $\mathrm{R}$ was used.

\section{Results}

The Bass models (Fig. 1) revealed distinct patterns of diffusion in German and Finnish hospitals. For Finland, the data showed a quick and almost similar uptake process across all three HIT systems. The models reflect this pattern through the high coefficients of imitation $(q)$ across the systems, albeit with slightly faster adoption of radiology systems which were introduced after EHRs. However, Finnish hospitals had achieved full adoption of RIS, PACS and electronic records in 2007.

In contrast, the Bass models mirrored a slower uptake in Germany compared to Finland. Germany had a higher p coefficient (classical understanding "innovation") but lacking "imitation" effects, as is indicated by the low coefficients q, eventually failing to reach full adoption as of 2021 (Tab. 1). One common feature of diffusion patterns in both countries was the slower adoption of electronic records compared to radiology systems, although this observation is less prominent in Finland (Tab. 1).

All curves showed a good model fit with no significant deviations (p-values, Tab. 1)

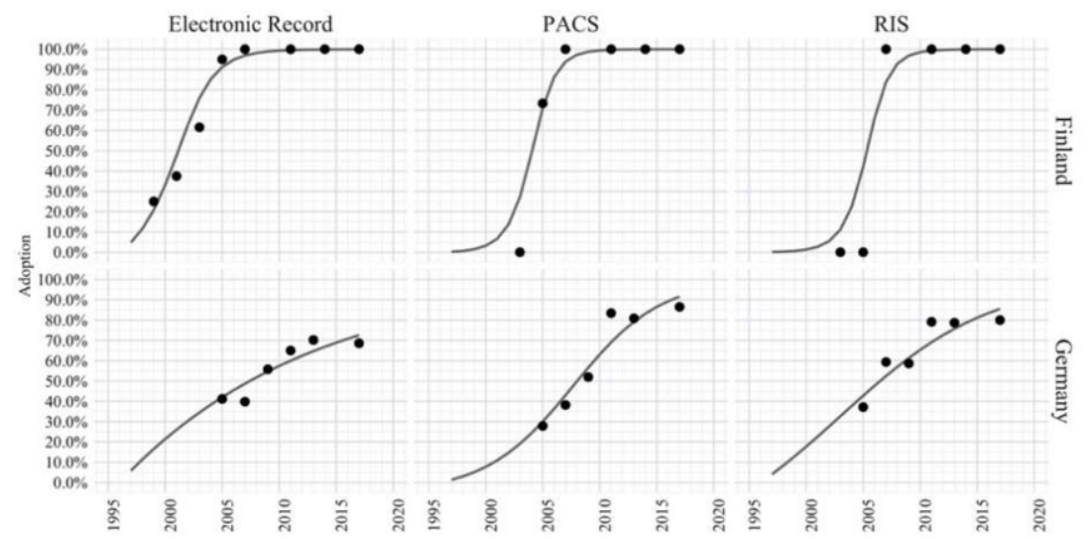

Figure 1. Observed adoption data (points) and the modelled diffusion curve for electronic records, PACS and RIS (lines) for Germany and Finland.

\section{Discussion}

We investigated the diffusion of three HIT systems in Finland and Germany. A key characteristic in the diffusion process was the higher coefficient of "innovation" in Germany than Finland, indicating the existence of initial innovators. But only a few peer institutions followed suit resulting in notably slower diffusion and failing the complete uptake of those three systems. The lack of "imitation" may also indicate missing network effects [5]. In contrast, Finland, for which the models showed high "imitation" effects witnessed quick gains in digitizing hospital care, mainly between 2005 and 2007 for RIS and PACS. This also held true for the more complex EHR systems.

The size of both countries can partly explain these findings in terms of inhabitants, which indicates that smaller countries have fewer difficulties in rolling out HIT systems 
nationwide. In addition, governance and financing structures, large-scale standardization and nation-wide coordinating functions for implementation can ease or hinder implementation efforts. However, the political environment in which decision-making and enforcement of these decisions occur could be even more important.

Table 1. Bayesian Bass Diffusion Models, the Goodness of Fit, and model forecasts of the adoption rate in 2021 with the $95 \%$ Highest Density Interval (in brackets).

\begin{tabular}{|c|c|c|c|c|c|c|c|}
\hline \multirow{2}{*}{ Country } & \multirow{2}{*}{ System } & \multicolumn{2}{|c|}{$\begin{array}{c}\text { Model } \\
\text { Coefficients }\end{array}$} & \multicolumn{3}{|c|}{ Goodness of Fit } & \multirow{2}{*}{$\begin{array}{c}\text { Predicted } \\
\text { Adoption } \\
2021\end{array}$} \\
\hline & & $\mathbf{p}$ & $\mathbf{q}$ & $\mathbf{C h i}^{2}$ & $\begin{array}{c}\text { p- } \\
\text { value }\end{array}$ & df & \\
\hline \multirow{3}{*}{ Germany } & RIS & 0.039 & 0.112 & 7.046 & 0.217 & 5 & $90.4 \%[86.9 \%-93.4 \%]$ \\
\hline & PACS & 0.012 & 0.250 & 6.799 & 0.236 & 5 & $95.9 \%[94.2 \%-97.5 \%]$ \\
\hline & EPR & 0.062 & 0.000 & 4.256 & 0.513 & 5 & $77.2 \%[71.7 \%-82.6 \%]$ \\
\hline \multirow{3}{*}{ Finland } & RIS & 0.001 & 1.036 & 6.500 & 0.261 & 5 & $100.0 \%[100.0 \%-100.0 \%]$ \\
\hline & PACS & 0.002 & 1.000 & 3.050 & 0.692 & 5 & $100.0 \%[100.0 \%-100.0 \%]$ \\
\hline & EHR & 0.041 & 0.580 & 0.650 & 0.999 & 7 & $100.0 \%[99.9 \%-100.0 \%]$ \\
\hline
\end{tabular}

In Finland, the MSAH is the highest decision-making authority that oversees the municipalities responsible for providing the care. Connected systems, e.g. a standardized EHR linked with additional services, were central to the Finnish national strategy, which could be enforced in a top-down attitude [11]. These systems are integrated into Kanta, the Finnish national data services, a crucial building block of the Finnish eHealth strategy. As early as 2007, the first phase of the Kanta roll-out started based on legislation with the MSAH in charge [12].

In Germany, the national health care system is governed by the Federal Joint Committee, a committee representing the stakeholders, i.e. health insurers, hospitals and physician associations, that decides on binding regulations putting health care acts into practice. These stakeholders failed to reach consensus on a health IT strategy, on applications and health IT standards, essentially leaving IT adoption to the free market for an extended period of time. Missing financial support in Germany may have impeded imitation in Germany. However, recently, parliament passed laws that provide funding to facilitate system adoption, particularly for the hospital sector [13].

As emphasized, the electronic records in Germany lack interoperability whereas Finland's national strategy embraced connected EHRs allowing health information exchange. The ability to share information across organizational silos leverage the benefits of HIT [14], which itself facilitates its adoption and use. This might further explain Finland's quicker uptake.

Against this backdrop, the interpretation of the Bass diffusion coefficients has to be different for the two countries. Whereas in Germany, the interpretation of "innovation" and "imitation" may be vindicated, in Finland, where no free market forces were driving the adoption, the coefficients call for a new interpretation. In particular, "imitation" has to be reinterpreted as a centrally supported roll-out process. This process appeared to have worked very well, also for the rather complex electronic record systems. 


\subsection{Limitation}

The main limitation is related to the different systems in Finland (EHR) and Germany (EPR) and their comparability. Only in Finland did these record systems really connect institutions. In Germany, the EPR is in most cases an intramural system. There were no data available on the adoption of patient records within the hospitals for Finland and virtually no data for true EHRs for Germany.

\subsection{Conclusion}

In conclusion, the Bass diffusion models revealed distinct characteristics of HIT diffusion patterns in Germany and Finland, with Finland showing a noticeably faster uptake and earlier nationwide diffusion. This might be explained in light of different governmental and strategic approaches. Consequently, the "imitation" coefficient needs to be interpreted anew as a measure to reflect the success and speed of the "roll-out" process in Finland. In order for Bass models to be adequately understood, the environment in which the diffusion process takes place has to frame the interpretation.

Acknowledgement.This study was funded by the Ministry of Science and Culture, State of Lower Saxony, Germany (grant no. ZN 3062, project INITIATIVE eHealth).

\section{References}

[1] WHO Global Observatory for eHealth and World Health Organization, Global diffusion of eHealth: making universal health coverage achievable: Report of the third global survey on eHealth. 2016.

[2] Sabes-Figuera R, Abadie F. Institute for Prospective Technological Studies, European hospital survey: benchmarking deployment of e-health services (2012-2013): country reports. Luxembourg: Publications Office, 2013.

[3] Ammenwerth E et al. International Comparison of Six Basic eHealth Indicators Across 14 Countries: An eHealth Benchmarking Study. Methods Inf Med. 2020 Dec;59(S 02):e46-e63.

[4] Pianykh OS, Digital imaging and communications in medicine (DICOM): a practical introduction and survival guide, 2nd ed. Heidelberg;New York: Springer, 2012.

[5] Esdar M, Hüsers J, Weiß JP, Rauch J, Hübner U. Diffusion dynamics of electronic health records: A longitudinal observational study comparing data from hospitals in Germany and the United States. Int $\mathrm{J}$ Med Inform. 2019 Nov; 131:103952.

[6] Vehko T, Routsalainen S, Hyppönen H, E-health and e-welfare of Finland - Check Point 2018, National Institute for Health and Welfare, Helsinki, Finland, 2019.

[7] Huang HK. PACS and Imaging Informatics: Basic Principles and Applications. Hoboken, NJ, USA: John Wiley \& Sons, Inc., 2004.

[8] Rogers EM, Diffusion of innovations, 5th ed. New York: Free Press, 2003.

[9] Bass FM, A New Product Growth for Model Consumer Durables, 1969 Manag. Sci.;15(5), 215-27.

[10] Hüsers J, Esdar M, Weiß JP, Hübner U. Diffusion Dynamics of Radiology IT - Systems in German Hospitals - A Bayesian Bass Model. Stud Health Technol Inform. 2019 Sep 3;267:11-19.

[11] Doupi P, Ruotsalainen P. eHealth in Finland: present status and future trends. Int J Circumpolar Health. 2004 Dec;63(4):322-7.

[12] V. Jormanainen, [Introduction of Kanta services between 2010 and 2014], 2015 Duodecim Laaketieteellinen Aikakauskirja, 131(13-14), 1309-17.

[13] Krankenhauszukunftsgesetz (KHZG), Bundesgesundheitsministerium, 2020. Available at https://www.bundesgesundheitsministerium.de/krankenhauszukunftsgesetz.html. Accessed Jan. 2021.

[14] Menachemi N, Rahurkar S, Harle CA, Vest JR. The benefits of health information exchange: an updated systematic review. J Am Med Inform Assoc. 2018 Sep 1;25(9):1259-65. 